\title{
Miszelle
}

\section{Die diskrete Planungsebene in Castel del Monte}

\author{
von Mehdi Nadj
}

\section{Überblick}

Castel del Monte wurde im Auftrag des Kaisers Friedrich II. in den 1240er Jahren in Apulien gebaut (Abb. 1). Da bereits in zahlreichen Monographien die einzigartige Architektur dieses Bauwerkes geschildert worden ist, verzichten wir hier auf seine Beschreibung und verweisen auf die vorhandenen Quellen. ${ }^{1}$ Die Bibliographie zur Architektur von Castel del Monte mit über 730 Publikationen weist zugleich auf das große Interesse der Forschung hin, das ,Geheimnis' dieses rätselhaften Bauwerkes zu enthüllen. ${ }^{2}$

Auf der Suche nach historischen Vorläufern einer abstrakten Maßart, die noch bis 1600 in Isfahan (Iran) angewandt wurde, ${ }^{3}$ stieß der Autor auf Castel del Monte und stellte fest, dass hier der Bauplan auf einer diskreten Planungsebene basiert, in der der formale Zahlenwert des Goldenen Schnitts als abstraktes Planmaß dient. Die Ursprünge dieser Maßart werden dann in der

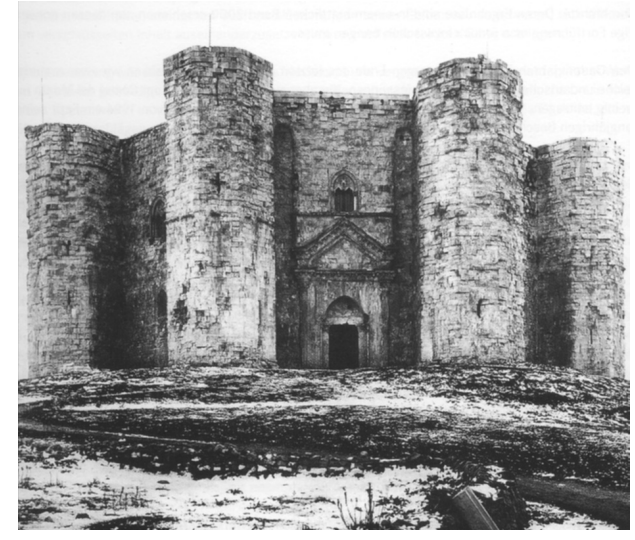

Abb. 1: Castel del Monte, historische Aufnahme. Quelle: Rolf Legler, Das Geheimnis von Castel del Monte, München 2008.

1 Heinz Götze, Castel del Monte, München 1991; Rolf Legler, Das Geheimnis von Castel del Monte, München 2008.

2 Dankwart Leistikow, Versuch einer Bibliographie zur Architektur von Castel del Monte, in: Oben und unten - Hierarchisierung in Idee und Wirklichkeit der Staufenzeit, Akten der 3. Landauer Staufertagung, Speyer 2005, S. 127-162; Jürgen Krüger, Bibliographie zur Architektur von Castel del Monte, in: Mythos Staufer, Akten der 5. Landauer Staufertagung, Speyer 2010, S. 157-158.

3 Mehdi Nadji, Normung nach geometrischer Progression im Isfahaner Normsystem, in: Ordo et Mensura 9, 2009, S. 1-8. 
antiken Bauplanung nachgewiesen. Die diskrete Planungsebene erlangt mittels Chiffrierung mystischer Zahlen eine sakrale Dimension. Die Übertragung des abstrakten Planmaßes auf das konkrete Baumaß basiert auf strengen Regeln, die stets zahlenästhetisch bestätigt werden. Diese Regeln wurden identifiziert und für die Rekonstruktion der im 19. Jahrhundert verschwundenen Wasserschale im Innenhof angewandt. Die Vermutung liegt nahe, dass Castel del Monte ein Prototyp für die Typisierung des Kastellbaus sein dürfte.

\section{Die geodätischen Grundlagen}

Die erste veröffentlichte Vermessungsarbeit von Castel del Monte stammt von Gino Chierici, der den neapolitanischen Palm als das Baumaß bestimmt

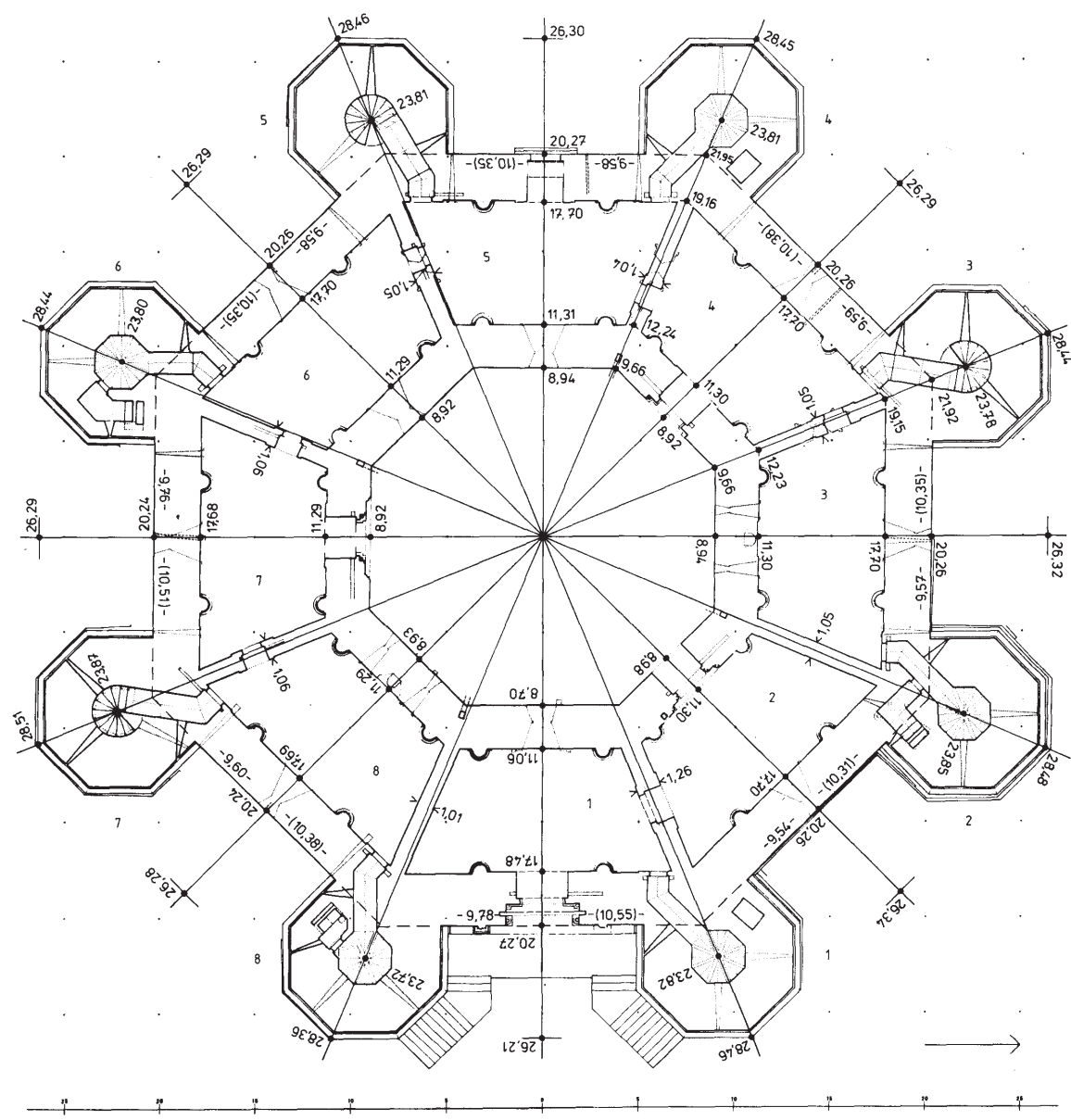

Abb. 2: Die wesentlichen Maße (m) im Erdgeschoss von Castel del Monte nach den Messungen der Schirmer-Gruppe. Quelle: Wulf Schirmer, Castel del Monte, Forschungsergebnisse der Jahre 1990-1996, Mainz 2000. 
hat. ${ }^{4}$ Später wurden systematische Abweichungen von diesem Maß festgestellt. ${ }^{5}$ Um eine Vielzahl von verschiedenen offenen Fragen über Castel del Monte zu klären, hat eine Forschergruppe um Wulf Schirmer das Bauwerk von 1990 bis 1996 systematisch untersucht. ${ }^{6}$ Von besonderer Bedeutung für unsere Untersuchung ist die Vermessungsarbeit dieser Gruppe, die - unter Berücksichtigung der Aufmaße Chiericis - von Wolfgang Zick durchgeführt worden ist (Abb. 2). ${ }^{7}$

Bei der Betrachtung der Abmessungen fällt auf, dass die in neapolitanischem Palm angegebenen Distanzen oft nicht plausibel bzw. überzeugend sind. Besonders auffallend ist etwa die angegebene Breite des trapezförmigen Raumes mit 24,33 Palm (Tabelle 1). Diese gebrochene Maßzahl entspricht nicht den üblichen planerischen Maßprioritäten; zumal das Bauwerk einzig diesen geschlossenen Raumtypus besitzt.

\begin{tabular}{|c|c|c|c|c|c|}
\hline & Distanz & $\begin{array}{l}\mathrm{Maß} \\
\text { (p) }\end{array}$ & $\begin{array}{l}\text { heit } \\
(\phi)\end{array}$ & $\Delta(\%)$ & Anmerkungen \\
\hline 1 & $\begin{array}{l}\text { Inkreisradius, } \\
\text { Brunnenschale }\end{array}$ & 6,50 & 4,0 & 0,4 & Berechnet (a in Abb. 12) \\
\hline 2 & $\begin{array}{l}\text { Brunnenrand- } \\
\text { Hofwand }\end{array}$ & 27,50 & 17,0 & 0,0 & Berechnet (b in Abb. 12) \\
\hline 3 & Hofwandstärke & 9,00 & 5,5 & 1,1 & Gemessen (BC in $\mathrm{Abb} .3)$ \\
\hline 4 & $\begin{array}{l}\text { Trapezraum- } \\
\text { breite }\end{array}$ & 24,33 & 15,0 & 0,2 & Gemessen (CD in Abb. 3 ) \\
\hline 3 & $\begin{array}{l}\text { Außenwand- } \\
\text { stärke }\end{array}$ & 9,73 & 6,0 & 0,2 & Gemessen (DE in Abb. 3) \\
\hline 6 & Turmradius & 16,27 & 10,0 & 0,5 & Gemessen (FG in Abb. 4) \\
\hline
\end{tabular}

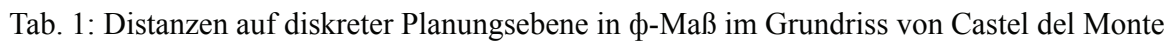

Da die metrologischen Ungereimtheiten Zick bewusst waren, ging er zunächst von einem etwaigen Fehlgriff bei der Maßeinheit aus. Um sich deren Richtigkeit zu vergewissern, überprüfte er alle relevanten Distanzen im Bauwerk

4 Gino Chierici, Castel del Monte, in: Monumenti Italiana, secolo XIII, Rom 1934.

5 Florian Huber, Jesi und Bethlehem, Castel del Monte und Jerusalem, in: Kunst im Reich Kaiser Friedrich II. von Hohenstaufen, München 1996, S. 45-51.

6 Wulf Schirmer, Castel del Monte, Forschungsergebnisse der Jahre 1990-1996 (in Zusammenarbeit mit Günter Hell, Ulrike Hess, Dorothée Sack, Werner Schnuchel, Christopher Uricher u. Wolfgang Zick mit Fotografien von Rafael Cardenas-Dopf), Mainz 2000.

7 Wulf Schirmer u. Wolfgang Zick, Castel del Monte, Zweiter Vorbericht, in: architectura 8, 1998, S. 1-36, hier S. 5; Schirmer (wie Anm. 6), S. 88. Der Turmradius MN errechnet sich aus der Abbildung 58 derselben Quelle. $\Delta=$ Differenz zwischen „Ist“ und „Soll“ von $\phi$-Werten. 


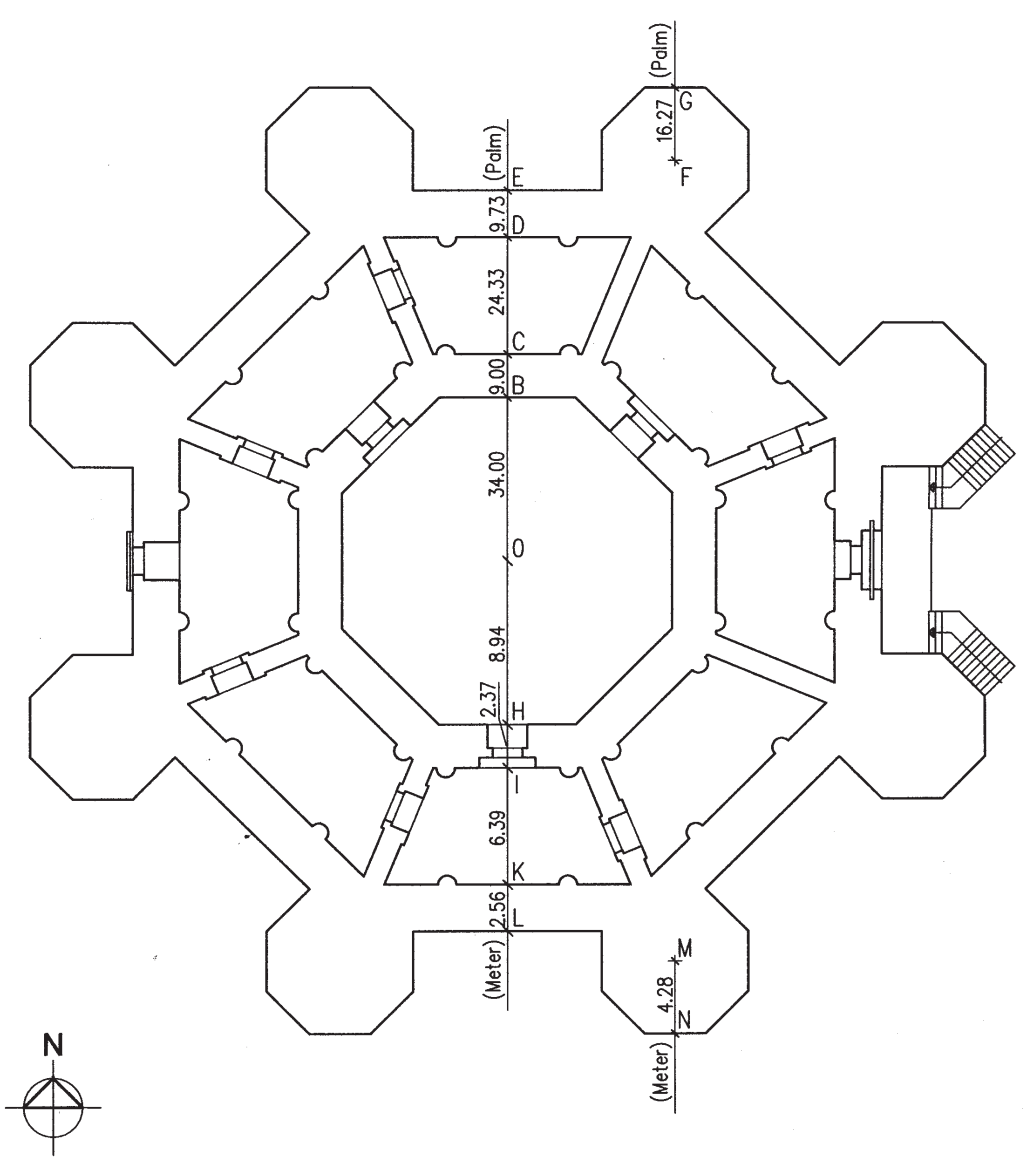

Abb. 3: Die Hauptachse des Grundrisses in Meter und Palm. Quelle: Eigene Zeichnung.

mit Maßlängen vom 0,260 bis 0,322 $\mathrm{m}$ in Millimeterschritten mit dem Ziel, die Häufigkeit der ganzzahligen Vielfachen zu bestimmen. Dabei erhielt er wiederum die beste Übereinstimmung mit $0,263 \mathrm{~m}$, d.h. neapolitanischem Palm. ${ }^{8}$ Dennoch war er mit der Maßfindung unzufrieden: „[Es] darf nicht verschwiegen werden, dass sich damit auch jetzt die maßlichen Verhältnisse von Castel del Monte noch nicht in jeder Hinsicht schlüssig haben erklären lassen.“9

\section{Divina proportio}

Vor diesem Hintergrund und mit Rücksicht auf die Vielzahl unbefriedigender Maßfindungsversuche anderer Autoren, schien es angebracht, das Problem mit

8 Ebd., S. 29.

9 Ebd., S. 33. 
einer abstrakten Maßart zu untersuchen. ${ }^{10}$ Denn wenn das Baumaß selbst auf der soliden Grundlage der äußerst präzisen Vermessungsarbeit von Wolfgang Zick nicht schlüssig festzustellen ist, kann nur noch ein abstraktes Maß in Frage kommen. In den vormetrischen Jahrhunderten konnte ein Baumeister von einer großen Auswahl an Maßeinheiten kreativ Gebrauch machen, so z.B. Module kreieren und damit ein Pseudo- bzw. abstraktes Maß anwenden. ${ }^{11}$ Vor diesem Hintergrund und aufgrund der Erfahrungen mit dem Isfahaner Normsystem wurden unterschiedliche formale Zahlenwerte des Goldenen Schnitts (Abb. 4) auf Distanzen im Castel del Monte getestet (Tab. 1).

Abb. 4: Minor und Major im Goldenen Schnitt. Quelle: Eigene Zeichnung
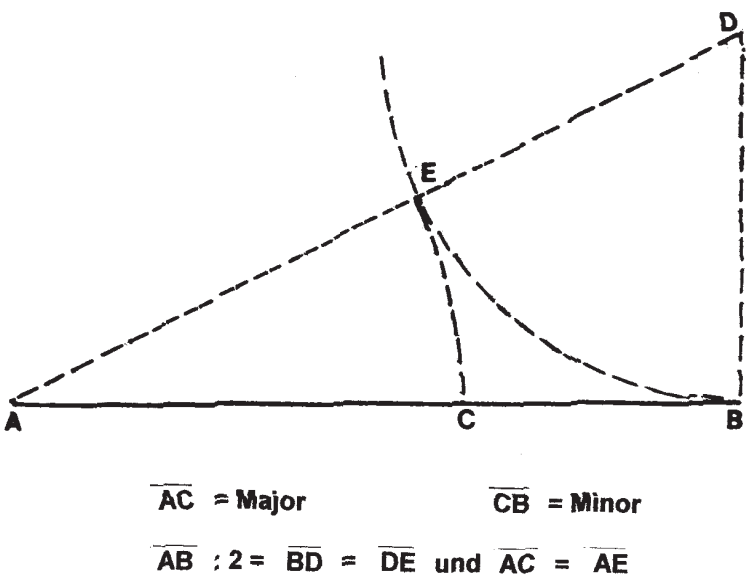

$\overline{\mathrm{AB}}: \overline{\mathrm{AC}}=\overline{\mathrm{AC}}: \overline{\mathrm{CB}}$

Es hat sich herausgestellt, dass alle in Palm angegebenen Distanzen ganzzahlig in dem formalen Zahlenwert des Goldenen Schnitts $(=1,618)$ aufgehen. ${ }^{12} \mathrm{Um}$ etwaige Zweifel auszuschließen, wurde die obige Prozedur mit anderen zur Diskussion stehenden Maßeinheiten (etwa Nippur-Elle, ägyptische Königselle, pes Romanus oder Gudea-Elle) wiederholt und dabei festgestellt, dass die neapolitanische Palm-Einheit $(=26,3 \mathrm{~cm})$ eindeutig das beste Ergebnis liefert. Da aber der Goldene Schnitt $(=\phi)$ als Zahlenverhältnis keine Dimension hat, muss er hier als ein ,flexibler Maßrahmen' betrachtet werden, der stets mit einer konkreten Maßeinheit ausgestattet sein muss. Dieser Rahmen wäre praktisch als ein variables ,Maßvehikel ‘ vorstellbar, das mit unterschiedlichen

10 Werner Heinz, Castel del Monte - zu Entwurf und Übertragung ins Gelände, in: Ordo et Mensura 6, 2000, S. 105-126, hier S. 106f.

11 Georgy Kepes, Modul Proportion Symmetrie Rhythmus, Brüssel 1966, S. 118ff.; Paul v. Naredi-Rainer, Architektur und Harmonie, Köln 1982, S. 129f.

12 Die Ausnahme mit halbzahligem ф-Wert wird noch im Abschnitt über lineare Optimierung begründet. 
Baumaßarten ausgestattet werden kann. ${ }^{13}$ Die benutzte Baumaßeinheit in Castel del Monte ist also der neapolitanische Palm, der eindeutig dem formalen $\phi$-Wert $(=1,618)$ mit regelhaften Vielfachen genügt.

Der Goldene Schnitt wird demnach in der Planung von Castel del Monte in zwei verschiedenen Versionen angewandt. Einmal als veritables Verhältnis zweier Distanzen in herkömmlichem Sinne und dann als ein formaler Zahlenwert $(\phi=1,618)$, der in der Tat ein abstraktes Maß mit ästhetischem Symbolgehalt ist. Hierfür lassen sich verschiedene Beispiele auch in der Architekturgeschichte finden, auf die wir noch eingehen werden (Abb. 4). Zusätzlich zu der assoziativ ästhetischen Qualität kommt dem Goldenen Schnitt in Castel del Monte eine weitere quasi sakrale symbolische Bedeutung zu; zumal er im Mittelalter divina proportio hieß. ${ }^{14}$ Wir werden diesen wichtigen symbolischen Aspekt des „,göttlichen Verhältnisses“ in der Kastellplanung noch näher beschreiben.

\section{Die diskrete Planungsebene als vierte Baudimension}

Während nun eine sichtbare Planungsebene durch die Vielzahl von ineinander verschachtelten und symmetrisch geordneten Oktogonalformen direkt wahrgenommen wird (Abb. 2), gibt es offensichtlich noch eine weitere Planungsebene, die als Matrix des abstrakten Maßrahmens fungiert, aber sich der direkten Anschauung entzieht. Diese verborgene bzw. diskrete Planungsebene wirkt sich durch metrologische Fakten aus und ist auch aufgrund ihrer metrologischkünstlerischen Attribute nachweisbar (Abb. 5).

$\mathrm{Zu}$ den metrologischen Fakten gehört zunächst die Tatsache, dass durch die Berücksichtigung der diskreten Planungsebene alle bislang offenen Baumaßfragen schlüssig und stimmig gelöst werden. Hierbei sind die Differenzen $(=\Delta)$ zwischen den Palm- und $\phi$-Zahlenwerten minimal (Tab. 1). Die für $\phi$ errechneten Maßzahlen sind - mit einer Ausnahme - ganze Zahlen. Dabei ist nicht zu übersehen, dass selbst bei dieser Notlösung ersatzweise für das Baumaß Palm eine ganze Maßzahl weiterhin besteht (Tab. 1).

Besonders stichhaltig ist die in der diskreten Planungsebene errechnete Breite des trapezförmigen Raumes von $\mathrm{CD}=15$ क; zumal sie das gemessene aber unpassend erscheinende Maß von 24,33 p neu interpretiert und als fehlerfreies Bauma $\beta$ identifiziert. Das Planungsma $\beta=15 \phi$ ist aber ganzzahlig und markant (Abb. 5). Dennoch ist der wichtigste Existenzbeweis für die diskrete Planungsebene die Chiffrierung mystischer Zahlen und ihre sakralen Bedeu-

13 Der Begriff „Modul“ wäre hier angebracht, wenn man davon ausgehen könnte, dass die Konstante $\mathrm{C}$, bei jedem Achteck $\mathrm{C}=0,063$, damals um ca. $2 \%$ ungenau und gleich 0,0618 , berechnet wäre. Dann ergäbe ihre Verzehnfachung den reziproken Wert von $\phi=1,618$. Die Konstante $\mathrm{C}$ ergibt sich aus $\mathrm{C}=(\mathrm{a}: \mathrm{r})-(\mathrm{a}: \mathrm{R})=0,063$, während nach mathematischem Tabellenwerk $\mathrm{a}: \mathrm{r}=0,8284$ und $\mathrm{a}: \mathrm{R}=0,7654$ ist. Dabei ist $\mathrm{a}=$ Oktogonseite, $\mathrm{r}=$ Radius des Inkreises und $\mathrm{R}=$ Radius des Umkreises.

14 Albrecht Beutelspacher u. Bernhard Petri, Der Goldene Schnitt, Mannheim 1995, S. 10. 


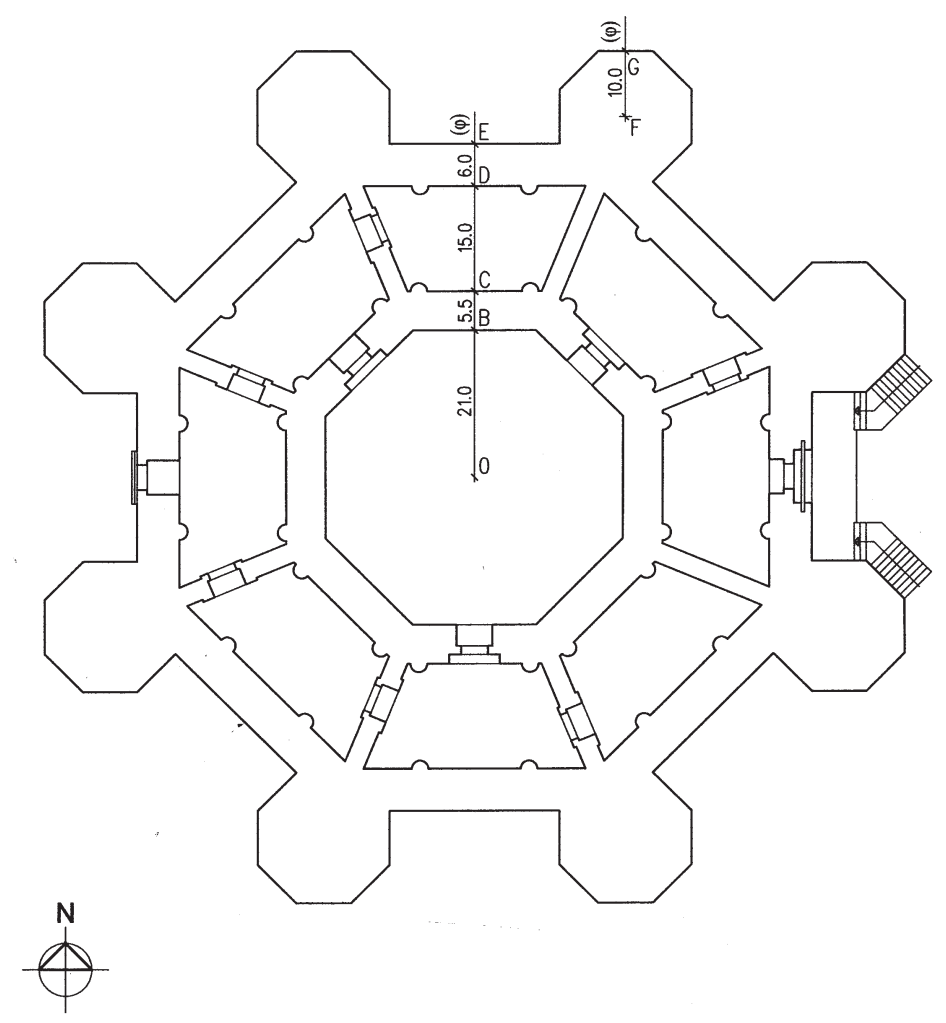

Abb. 5: Die diskrete Planungsebene in abstrakter Maßeinheit $\phi=1,618$. Quelle: Eigene Zeichnung.

tungsinhalte, womit wir uns noch beschäftigen werden. Die ausführliche Diskussion darüber, ob die diskrete Planungsebene als eine vierte Dimension im Bauplan angesehen werden kann, würde den Rahmen dieser Arbeit sprengen.

\section{Planungstiefe und Zahlenästhetik}

Ein besonderes planerisches Kriterium von Castel del Monte ist seine Planungstiefe, die bei Anschauung des Bauwerkes und ohne Kenntnis der Details der diskreten Planungsebene nicht festzustellen ist. Der Kürze wegen verzichten wir hier auf detaillierte Zahlentabellen und versuchen mit nur wenigen planimetrischen Angaben die bemerkenswerte Planungstiefe und Zahlenästhetik in Castel de Monte anzudeuten. Ein aufschlussreiches Beispiel dafür findet sich unten im Abschnitt „Rekonstruktion der Brunnenschale“.

Grundsätzlich ist die Grundrissgliederung die Basis für die Symmetrieordnung des räumlichen Erscheinungsbildes, das vom Baumaß geprägt wird. Doch diese Gliederung erfüllt zugleich auch die Anforderungen des $\phi$-Schemas in der diskreten Planungsebene. In diesem Schema haben etwa die Flächengrößen 


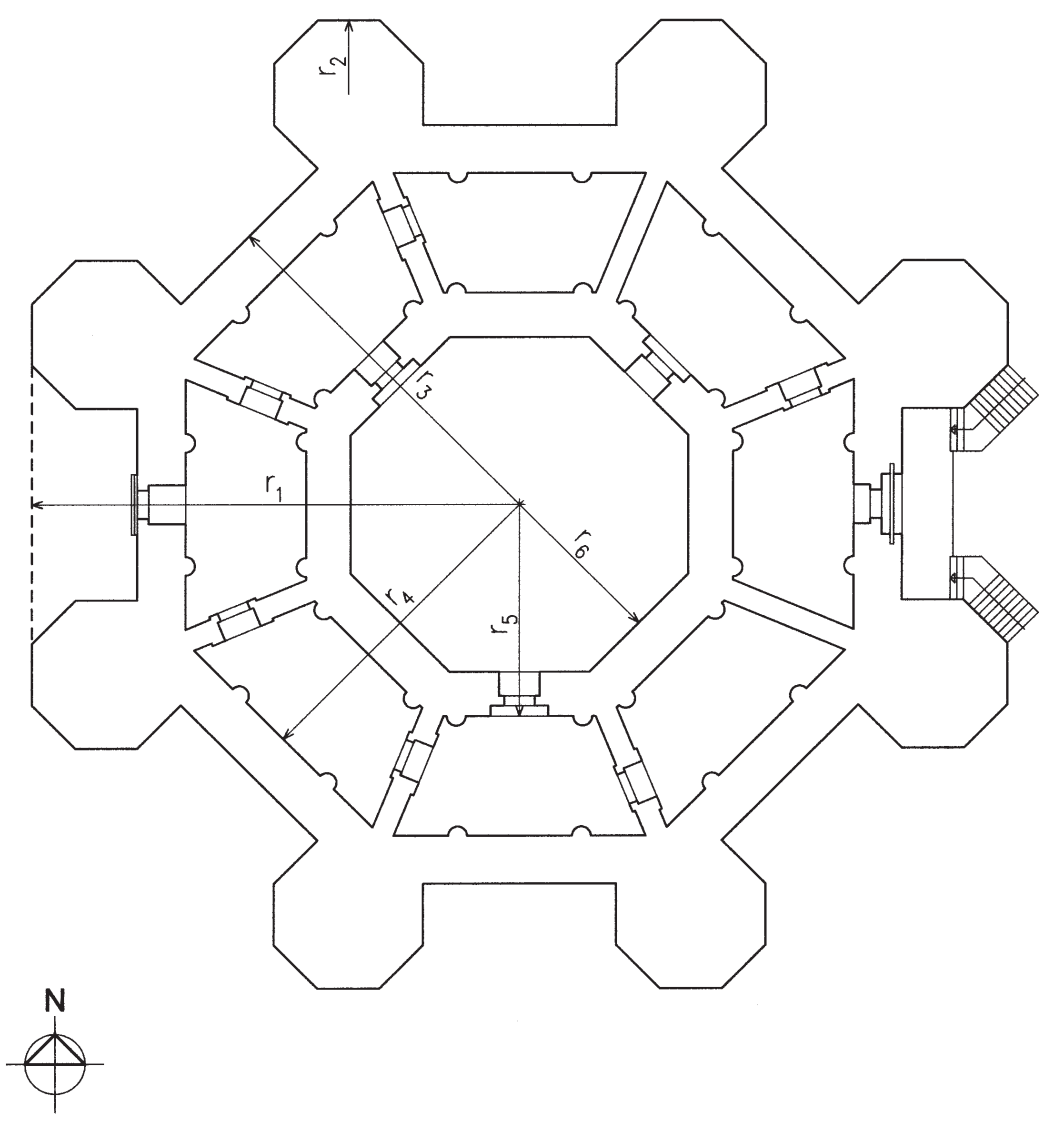

Abb. 6: Bezeichnung der Inkreisradien der Oktogone im Grundriss. Quelle: Eigene Zeichnung.

von fünf Achtecken(Abb. 6) ${ }^{15}$ sowie die ursprüngliche Trapezfläche F, jeweils mindestens ein definierbares bzw. prägnantes Zahlenverhältnis zueinander, das auch aus der $\phi$-Reihe stammen kann. ${ }^{16}$ Die bei dieser Verknüpfung fehlenden zwei Achteckflächen (von $\mathrm{r}_{3}$ und $\mathrm{r}_{4}$ in Abb. 6) sind offensichtlich für die Erfordernisse der linearen Optimierung der Trapezfläche geändert worden.

Die gegenwärtige Trapezraumfläche beträgt $404 \mathrm{Phi}^{2}$ und hat keine metrologische Verbindung zu den übrigen Grundrissformen. ${ }^{17}$ Auch lässt sich ihre Fläche nicht zahlenmystisch interpretieren. Die Trennwandstärken sind aber so gewählt,

15 Es sind Oktogone mit den Inkreisradien $r_{1}, r_{2}, r_{5}, r_{6}$ und $r_{7}$ (Abb. 6).

16 Die geometrische Reihe des Goldenen Schnitts $\phi$ lautet:
$\ldots 0,382$
0,618
1
1,618
2,618
4,236
$\begin{array}{llllllll}\ldots & \phi^{-2} & \phi^{-1} & 1 & \phi & \phi^{2} & \phi^{3} & \ldots\end{array}$
$\begin{array}{llllllll}\ldots & \phi^{-2} & \phi^{-1} & 1 & \phi & \phi^{2} & \phi^{3} & \ldots\end{array}$
$\ldots=$

$17 \phi^{2}(=2,618)$ befindet sich in der geometrischen Reihe des Goldenen Schnitts (siehe Anm. 16) und ist eine dimensionslose Zahl, die zahlenmystisch interpretiert werden darf. Dagegen stellt $\phi \times \phi=\phi^{2}$ eine abstrakte Flächeneinheit bzw. eine „Dimension“ dar, die bei der 
dass nun aus dem Verhältnis beider Trapezgrundlinien ein Goldener Schnitt entsteht (Abb. 7). Bei der ursprünglichen Trapezfläche $\left(=423,6 \mathrm{Phi}^{2}\right)$ können neben der Differenzierung durch mystische Zahlensymbolik auch die folgenden Kunstfertigkeiten festgestellt werden. Die Raumbreite bzw. Trapezhöhe mit $\mathrm{H}$ $=15 \phi$ (Abb. 5) erweist eine zahlenmystische Beziehung zu Maria. Denn die Zahl 15 (=3 x 5) steht für die Trinität und auch für die Marienzahl 5; zumal im Neuen Testament fünf Marien erwähnt werden. Außerdem hat die 5 auch den Rhythmus der Marienfeste bestimmt. ${ }^{18}$ Ferner betragen die Grundlinien der ursprünglichen Trapezform 34,5 ф bzw. 22 , wobei ihr Verhältnis ebenfalls dem veritablen Goldenen Schnitt nahe kommt:

$34,5: 22=1,57(\text { Differenz zu } \phi<3,0 \%)^{19}$

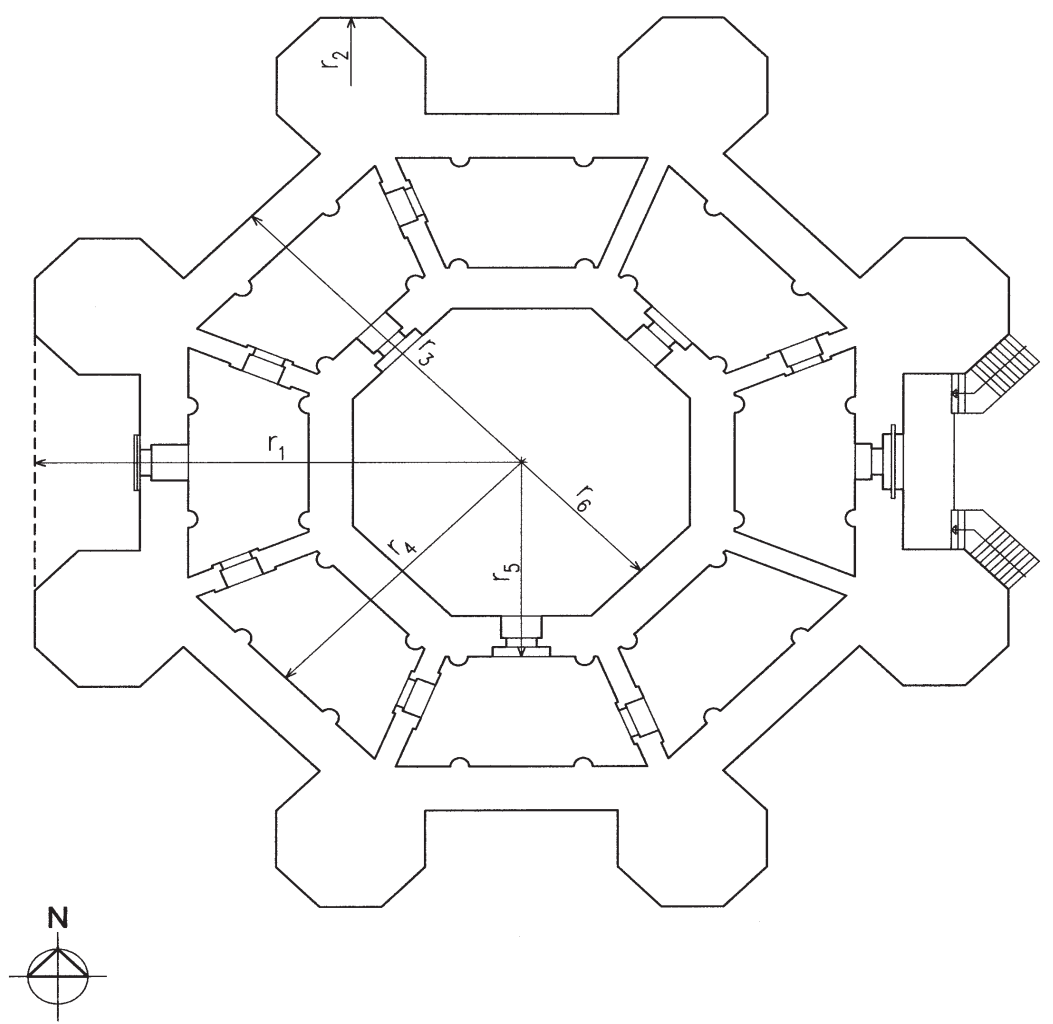

Abb. 7: Grundriss mit Trennwänden, Maße in Meter, nach den Messungen der Schirmer-Gruppe. Quelle: Eigene Zeichnung

zahlenmystischen Berechnung nicht berücksichtigt wird; zumal im 13. Jahrhundert eine konsequente Dimensionierung noch nicht üblich war.

18 Franz Endres u. Annemarie Schimmel, Das Mysterium der Zahl, Köln 1984, S. 124.

19 Abweichungen $(=\Delta)$ werden nach Albrecht Kottman und auch M.S. Bulatov bis $3 \%$ toleriert. Vgl. Heinz Götze, Die Baugeometrie von Castel del Monte, Heidelberg 1991, S. 38. 
Diese Attribute sind als Randbedingungen zusätzlich bei der Lösung der linearen Optimierung der Trapezfläche berücksichtigt worden.

Folgerichtig sind auch die Raumhöhen durch christliche Zahlenmystik ausgezeichnet. Damit ist dem gesamten Innenraum der Burg metrologisch eine Aura der Frömmigkeit verliehen worden. Die ausführliche Beschäftigung mit diesem Fragenkomplex würde aber eine eigene Abhandlung erfordern.

Nun steht diese Architektur des stillen Gebetes vordergründig im Widerspruch zu dem oft dargestellten Persönlichkeitsbild Kaiser Friedrich II., das mit prägnanten politischen Kampagnen gegen den Papst charakterisiert wird. Doch dieser Zwiespalt ist, wie Schaller beweist, rein äußerlich und wird behoben, wenn man bei der Religiosität des Herrschers zwischen seiner persönlichen Frömmigkeit und seiner Kirchenpolitik sehr genau unterscheidet. ${ }^{20}$

Auf die hier festgestellte durchdachte Planungsmethode werden wir im Abschnitt „Rekonstruierung der Brunnenschale“ zurückkommen.

\section{Optimierung der Trapezfläche}

Die Analyse der Planungstiefe hat gezeigt, dass der Bauplan durch zahlenästhetische Attribute kunstvoll sublimiert worden ist. Die Vermutung liegt also nahe, dass der Baumeister das profane Palm-Maß in die divina proportio transferiert hat ${ }^{21}$ um mit diesem weihevollen Pseudomaß u.a. auch imaginäre Bauelemente zu gestalten, die in architektonischer Sprache religiöse Überzeugungen und fromme Sentenzen zum Ausdruck bringen. Diese Vermutung wird schließlich durch die Berechnung der ursprünglichen Trapezfläche bestätigt. Denn der trapezförmige Raum (ohne Trennwände) hat nach Abb. 8 die Grundfläche

$$
\begin{aligned}
& \mathrm{F}_{\mathrm{t}}=\mathrm{H}(\overline{\mathrm{AB}}+\overline{\mathrm{CD}}): 2 \\
& \text { d.h. } \mathrm{F}_{\mathrm{t}}=15(34,5+22,0): 2=423,75 \mathrm{Phi}^{2} .
\end{aligned}
$$

Bei Vernachlässigung einer Differenz von $\Delta=0,03 \%$ kann diese Flächenzahl mit 423,6 gleich gesetzt und umgeformt werden in

$$
423,6=4,236 \times 100 .
$$

Auf die Interpretation der überaus markanten Ziffernfolge 4,236, die uns aus der geometrischen Reihe des Goldenen Schnitts bekannt ist, ${ }^{22}$ kommen wir später zurück, stellen aber zunächst fest, dass diese Ziffernkombination nur durch gezielte und aufwändige Optimierungsrechnungen zustande gekommen sein kann (Abb. 8). Bei diesem Optimierungsproblem ist die Flächenzahl von 423,6 im Voraus festgelegt worden. Dann bestand die Aufgabe darin, die Ab-

20 Hans Martin Schaller, Die Frömmigkeit Kaiser Friedrich II., in: Deutsches Archiv für Erforschung des Mittelalters 51, 1995, S. 593-613, hier S. 594f.

21 Luca Pacioli beschreibt in seinem Buch „divina proportio“ (1509) 13 „wunderbare“ Eigenschaften des Goldenen Schnitts und führt sie auf die zwölf Apostel plus Jesus zurück; vgl. Le Corbusier, Modulor 2, Stuttgart 2003, S. 70.

22 Vgl. Anm. 16. 
Abb. 8: Lineare Optimierung der Trapezfläche. Quelle: Eigene Zeichnung

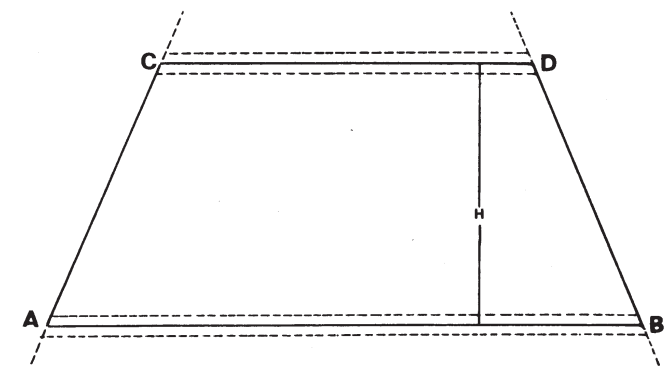

weichungen des Trapezgrundlinienverhältnisses $\Delta$ vom veritablen Goldenen Schnitt $(=1,618)$ so zu optimieren, dass $\Delta<3 \%$ bleibt. ${ }^{23}$ Außerdem sind noch folgende Nebenbedingungen zu berücksichtigen gewesen:

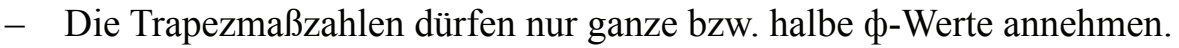

- Sie sollen auch eine bautechnisch erforderliche Mauerstärke ermöglichen.

Wir überprüfen die realisierten Ergebnisse und stellen fest, dass die Aufgabe befriedigend gelöst worden ist. Denn das Verhältnis beider Trapezgrundlinien errechnet sich als

$\overline{\mathrm{AB}}: \overline{\mathrm{CD}}=34,5 \phi: 22 \phi=1,57$ und $1,618-1,57=0,048$

Daraus ergibt sich ein $\Delta$-Wert von $2,96 \%$ und damit $<3 \%$.

Weiterhin ist die Berücksichtigung der ersten Prämisse bereits aus Abb. 5 ersichtlich. Im Falle der zweiten Bedingung hat man offensichtlich aus Verteidigungsgründen die ursprüngliche Stärke der Außenmauer beibehalten und nur die Hofmauerstärke auf 9 Palm $(=5,5 \phi)$ reduziert $(\Delta=1 \%)$. Die metrologische Verkettung dieser Mauerstärke im Rahmen der Planungstiefe wird bei der Rekonstruktion der Brunnenschale gezeigt.

Aus mathematischer Sicht wäre die Annahme berechtigt, dass das Optimierungsproblem in Castel del Monte mit Hilfe der Iteration gelöst worden ist. Grundsätzlich bleibt aber die Lösung und zugleich die Anwendung des Verfahrens im 13. Jahrhundert für die Mathematikgeschichte ein Novum; zumal die mathematisch exakte Lösung der Linearen Optimierung erst 1947 durch das Simplex-Verfahren möglich wurde. ${ }^{24}$

\section{Salutatio angelica}

„Das mittelalterliche Maß war qualitative und quantitative Größe in einem“, stellt Harald Witthöft fest und ergänzt, ,das Maß war zugleich Realität, Meta-

23 Vgl. Anm. 19.

24 Heinrich Bader u. Siegbert Fröhlich, Einführung in die Mathematik für Volks- und Betriebswirte, München 1968, S. 175. 
pher und Symbol innerhalb des mittelalterlichen ordo" ${ }^{25}$ In Castel del Monte erlangt das Maß auch eine sakrale Wertigkeit.

Wir haben gesehen, dass sich die Flächenzahl 423,6 auch als 4,236 x 100 ausdrücken lässt. Nun ist uns aber die Zahl 4,236 aus der geometrischen Reihe des Goldenen Schnitts bekannt. ${ }^{26}$

$$
4,236=1,618 \times 1,618 \times 1,618=\phi \times \phi \times \phi
$$

symbolisiert die Trinität. Da im Oktogon acht solche Trapezflächen im Rundgang vorhanden sind, gilt:

$8 \times 4,236=33,88$.

Diese Zahl, aufgerundet auf 34, symbolisiert die irdischen Lebensjahre Christi, die zwischen 33 und 34 Jahre angegeben werden. ${ }^{27}$ Der Rundzahl 100 kommt sowohl in ästhetischer als auch in sakraler Hinsicht große Bedeutung zu. Wir betrachten hier speziell ihre zahlenmystische Bedeutung und interpretieren sie als Zahl der Himmelskönigin Maria. ${ }^{28}$ Anders als die Marienzahl 5, die auf biblischen Quellen basiert, ${ }^{29}$ bezieht sich die Marienzahl 100 auf das Kirchengebet salutatio angelica. ${ }^{30}$

Zur Vorgeschichte des Ave-Maria-Gebets und seiner Verbindung zu der Rundzahl 100 schreibt Fritz Tschirch: „Erkennen lässt sich immerhin, dass das Ave verhältnismäßig spät, im Laufe des 11. und 12. Jahrhunderts, zu einem allgemeinen und häufiger benutzten Gebet wird und im ersten Viertel des 13. Jahrhunderts geradezu schlagartig zu seiner nun allerdings überwältigenden Beliebtheit aufschießt" ${ }^{\text {". }}{ }^{31}$ Das Geheimnis dieser überwältigenden Beliebtheit ist die Zahl der Buchstaben des Ave-Gebetes, die in mittelalterlicher Orthographie gleich 100 ist. ${ }^{32}$ Die Zahl 100 hatte für den mittelalterlichen Menschen neben ihrem runden und zentralen Wert auch eine mystische Bedeutung. „Hundert Buchstaben umfasste der englische Gruß“, konstatiert Edward Schröder. ${ }^{33}$ Somit legt Castel del Monte auch vom „schlagartigen Aufschießen“ des Ave-Gebetes im ersten Viertel des 13. Jahrhunderts Zeugnis ab.

25 Harald Witthöft, Maßgebrauch und Messpraxis in Handel und Gewerbe des Mittelalters, in: Albrecht Zimmermann (Hg.), Mensura, 1. Halbband 1983, S. 234-260, hier S. 234.

26 Vgl. Anm.16.

27 Fritz Tschirch, Die Bedeutung der Rundzahl 100 für den Umfang mittelalterlicher Dichtungen, in: Oskar Söhngen Festschrift, Gestalt und Glaube, Berlin 1960, S. 77-88, hier S. 79, sowie Heinz Mayer u. Rudolf Suntrup, Lexikon der mittelalterlichen Zahlendeutungen, München 1987, S. 704.

28 Tschirch (wie Anm. 27), S. 80.

29 Endres/Schimmel (wie Anm. 18), S. 72 ff.

30 Tschirch (wie Anm. 27), S. 80.

31 Ebd., S. 82.

32 Der Engelsgruß hieß im Wortlaut: „Ave Maria, gracia plena, dominus tecum, benedicta tu in mulieribus, et benedictus fructus ventris tui, Jhesus Cristus, Amen."

33 Tschirch (wie Anm. 27), S. 82. 


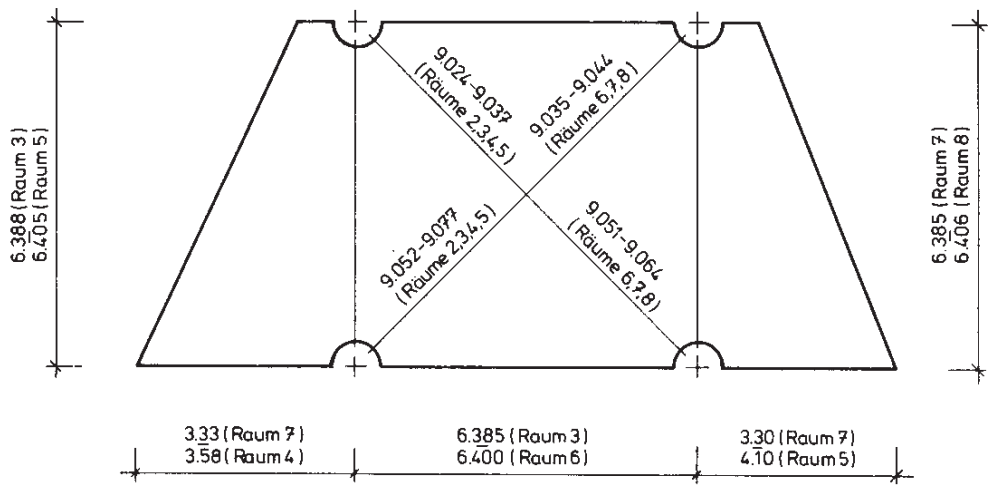

Abb. 9: Distanzen verschiedener Bauelemente in den Trapezräumen im Erdgeschoss nach den Messungen der Schirmer-Gruppe. Quelle: Eigene Zeichnung.

\section{Die Raumtrennwände}

Gänzlich unerwartet haben wir bei der metrologischen Analyse der Trapezraumflächen einen eindeutigen Hinweis darauf bekommen, dass die Raumtrennwände im ursprünglichen Bauentwurf nicht geplant waren. Da dieser Befund für die historische Entwicklung des Bauwerkes von besonderer Bedeutung ist, wird er im Folgenden auch durch andere Beweise weiter bekräftigt.

In der Fachliteratur finden wir kaum einen mittelalterlichen Profanbau, der in der Folgezeit nicht mindestens einmal eine Umgestaltung erfahren hat. Besonders betroffen sind davon die Burgen des 11. Jahrhunderts, die „die Hauptphase des deutschen Burgbaus" darstellen. ${ }^{34}$ Aber bereits im 14. Jahrhundert stiegen die Ansprüche hinsichtlich eines differenzierten und behaglichen Wohnens. ${ }^{35}$ Über die vielseitigen Änderungen an staufischen Profanbauten in Süditalien berichtet auch Carl A. Willemsen ausführlich, geht aber - wie andere Autoren - auf diese Frage für Castel del Monte nicht ein. ${ }^{36}$

Aus den Messdaten der Abbildung 9 lassen sich unterschiedliche Maßhaltigkeiten der Bauausführungen in den trapezförmigen Räumen erkennen. Wir betrachten die Spannweite R als Ausdruck der Maßhaltigkeit. Die Spannweite $\mathrm{R}$ ist die Differenz zwischen der größten und der kleinsten Länge eines bestimmten Bauelementes in verschiedenen Trapezräumen. Bei den ursprünglich geplanten Halbsäulenabständen $(\mathrm{R}=0,017 \mathrm{~m})$, aber auch bei den Trapezbreiten

34 Hans-Martin Maurer, Burgen, in: Die Zeit der Staufen, Bd. 3, Stuttgart 1977, S. 121.

35 Karl-Heinz Clasen, Die gothische Baukunst, Potsdam 1930, S. 212.

36 Carl A. Willemsen, Die Bauten Kaiser Friedrich II. in Süditalien, in: Die Zeit der Staufen, Bd. 3, Stuttgart 1977, S. 150. 
im Erdgeschoss $(\mathrm{R}=0,021 \mathrm{~m})$ sind die Spannweiten auffallend gering und zeugen von einer merklichen Durchführungsgenauigkeit (Abb. 9). Dagegen sprechen die durch die Trennwände entstandenen Wandfluchtlängen mit Spannweiten von $\mathrm{R}=0,84$ bzw. $\mathrm{R}=0,90$ für einen planlosen Mauerbau, der in einer späteren Zeit mit niedrigeren handwerklichen Fähigkeiten durchgezogen sein dürfte.

Als häufigste Fehler dieser Trennmauer werden genannt: verschwenkt, verdreht, versetzt bzw. verschoben und variierende Mauerstärke und oft nicht achsmittig. ${ }^{37} \mathrm{Im}$ Erdgeschoss sind wenige und im Obergeschoss ist in keinem Fall eine exakt achsmittige Zwischenwand festzustellen. ${ }^{38}$ Und es wird bemerkt, dass „die raumtrennenden Wände im Erd- und Obergeschoss oftmals nicht übereinander stehen. “39 Aus baustatischen Überlegungen wäre aber in einer sachkundigen Planung erforderlich, die Trennmauer im Obergeschoss exakt über diejenigen des Untergeschosses zu setzen. Diese vielfältigen Baudurchführungsmängel stehen im unübersehbaren Kontrast zu den ursprünglich errichteten Bauteilen, die kaum nennenswerte planerische oder handwerkliche Mängel aufweisen.

Weitere Unterstützung erhält die Hypothese des späteren Trennmauerbaus durch Überlieferungen, wonach viele Hochzeitsfeste von Rang, wohl beginnend mit der Vermählungsfeier Violantes (1249), des Kaisers legitimer Tochter, in Castel del Monte veranstaltet wurden. ${ }^{40}$ Schließlich dürfte die Hochzeit von Alfons I. von Aragón und Isabelle del Balzo 1487 in Castel del Monte ${ }^{41}$ darauf hindeuten, dass die Räume bis dahin noch nicht getrennt waren.

In der Tat hat der Besucher in Castel del Monte oft den Eindruck, sich in einem Labyrinth zu befinden (Abb. 10). Diese Wahrnehmung ist bei einigen Autoren so stark gewesen, dass sie die gegenwärtige labyrinthische Zugänglichkeit der Räume als ursprünglich konzeptionelles Planungsziel angesehen haben. ${ }^{42}$

Diese Auffassung, die nun als Fehleinschätzung gelten dürfte, zeigt zugleich die komplizierten Raumverbindungen im Kastell, woraus man schließen kann, dass die gegenwärtigen Räumlichkeiten für größere festliche Veranstaltungen nicht geeignet waren. Denn die einheitlich trapezförmigen

37 Schirmer/Zick (wie Anm. 7), S. 11f.

38 Ebd., S. 12.

39 Wulf Schirmer, Castel del Monte, neue Forschungen zur Architektur Kaiser Friedrichs II. (in Zusammenarbeit mit Günter Hell, Ulrike Hess, Dorothée Sack und Wolfgang Zick), in: architectura 24, 1994, S. 185-224, hier S. 195.

40 Ekkehart Rotter, Apulien, Köln 2000, S. 179. Außerdem wird von weiteren Hochzeitsfeiern im 14. Jh. berichtet.

41 Birgit Wagner, die Bauten des Staufenkaisers Friedrich II. - Monumente des Heiligen Römischen Reiches, Würzburg 2003, S. 64.

42 Ebd., S. 121f.; Cord Meckseper, Castel del Monte - Seine Voraussetzungen in der nordwesteuropäischen Baukunst, in: Zeitschrift für Kunstgeschichte 33, 1970, S. 211-231, hier S. 225 . 


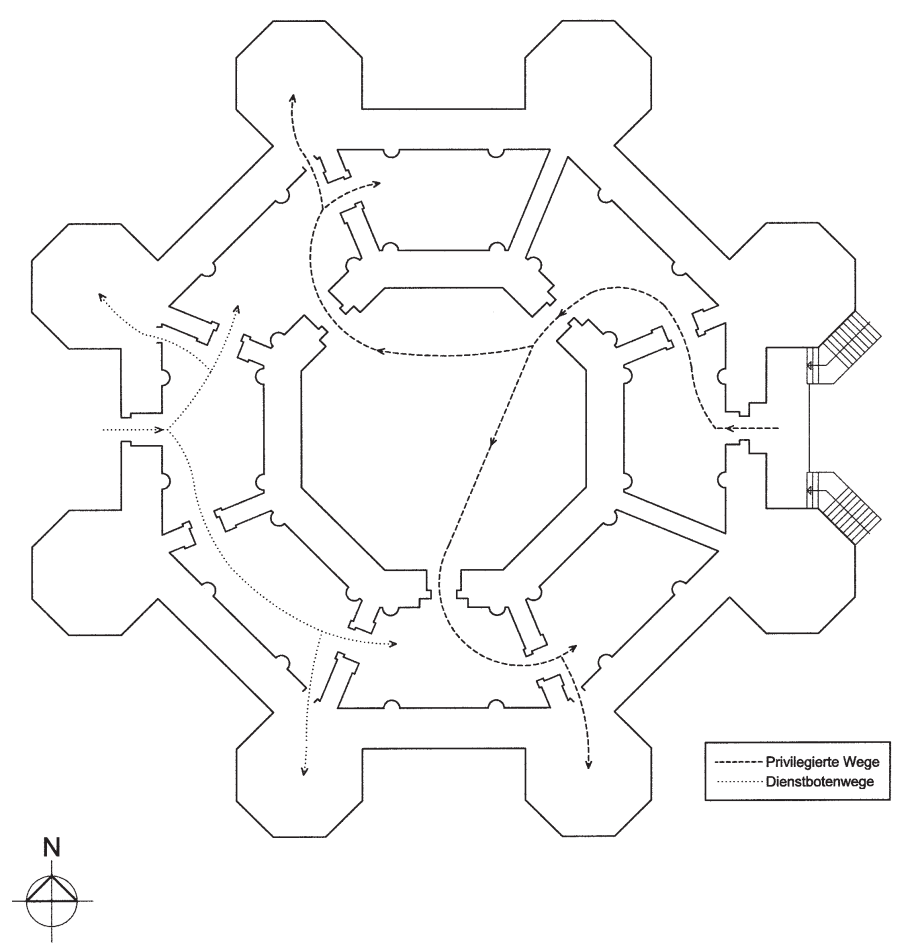

Abb. 10: Gegenwärtige labyrinthische Raumzugänge im Erdgeschoss. Quelle: Eigene Zeichnung.

Räume besitzen jeweils ca. 70 qm Grundfläche, auf der kaum mehr als zwei Dutzend erlesene Gäste zu bewirten gewesen wären. Dabei wäre auch kaum Raum für zusätzliche Spielleute geblieben. Schließlich war die mittelalterliche Hochzeitsfeier die Gelegenheit par excellence, den gesellschaftlichen Stand, Werte, Privilegien sowie Macht und Freigiebigkeit zur Schau zu stellen. ${ }^{43}$ Die Bevorzugung des Kastells für große Feierlichkeiten beruhte sicherlich auf seinem großen Raumangebot vor der Errichtung der Trennwände. Damals hat die Existenz großer Räume im ungehinderten Rundgang von ca. 600 qm je Stockwerk auch den Nachteil überdeckt, der durch die weit abgelegene Lage des Kastells gegeben war. Aus baustatischer Sicht würde die Annahme fehlender Trennwände in dem ursprünglichen Bauplan die Stabilität der Deckenkonstruktion nicht beeinträchtigen. Denn die Deckenkonstruktion basiert auf einem Kreuzrippengewölbe, das mit beidseitig anschließenden Spitzbogen-Tonnen über der Rundfläche kombiniert ist. ${ }^{44}$

43 Jacques Heers, Vom Mummenschanz zum Machttheater, Europäische Festkultur im Mittelalter, Frankfurt a.M. 1986, S. 20.

44 Schirmer/Zick (wie Anm. 7), S. 21. 


\section{Rekonstruktion der Brunnenschale}

Verschiedene Autoren berichten von einer Brunnenschale im Innenhof, die aufgrund ihrer Zerstörung bereits im 19. Jahrhundert beseitigt worden ist. ${ }^{45}$ In der Tat fällt es auch im Grundrissplan besonders deutlich auf, dass ein zentral komplementäres Element der Gesamtkonzeption nicht mehr vorhanden ist. „Die Leere des Innenhofs lässt einen heute den horror vacui anwandeln“, empfindet Carl A. Willemsen. ${ }^{46}$

Für die Rekonstruktion der ursprünglichen Brunnenschale versuchen wir zunächst unter Berücksichtigung der planimmanenten Regel die Schalenabmessungen zu bestimmen. ${ }^{47}$ Aufgrund des oktogonalen Formkonzepts des Bauwerks setzen wir als Schalenform ein Achteck voraus. ${ }^{48}$ Danach haben

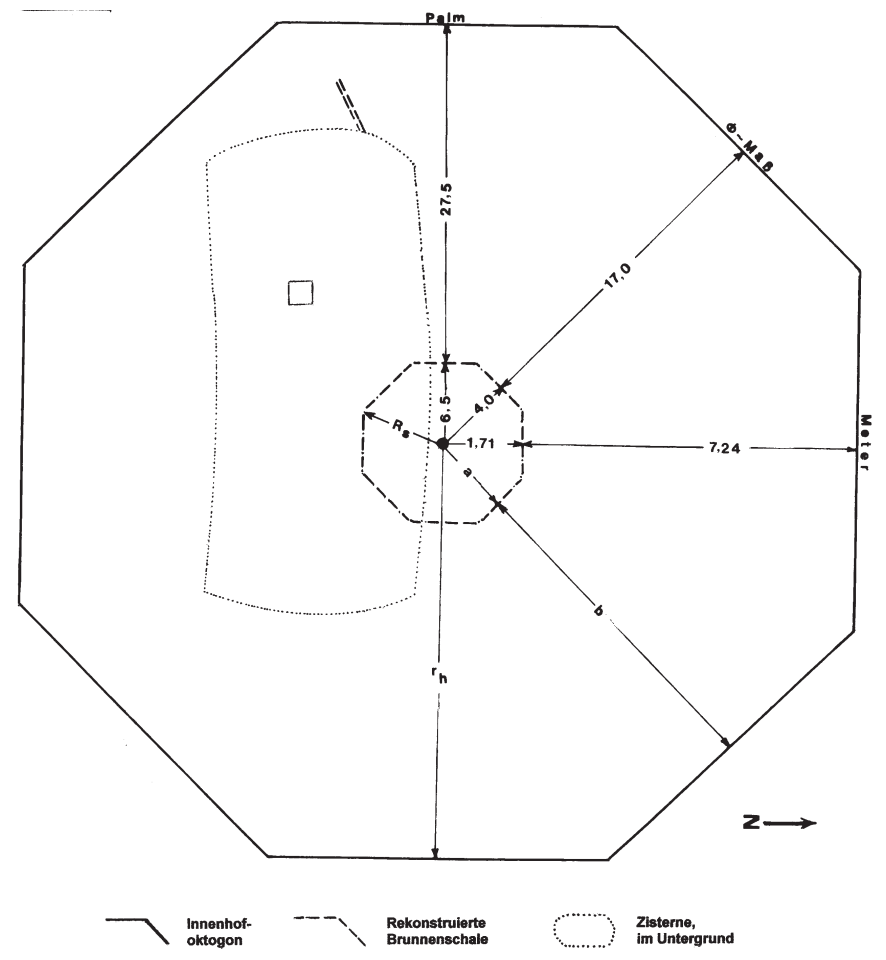

Abb. 11: Rekonstruktion der Brunnenschale. Quelle: Eigene Zeichnung.

45 In einigen Quellen wird von einem „Wasserbecken“ berichtet. Für den Bau eines Beckens muss aber der Boden ausgehoben werden, der wegen der Zisterne im Untergrund nicht vertieft werden darf (Abb. 11). Vgl. Legler (wie Anm. 1), S. 13.

46 Carl A. Willemsen, Castel del Monte, Frankfurt a.M. 1982, S. 72.

47 „Ganze Zahlen tragen zur Harmonie bei“ und ,jede Maßzahl hat mindestens eine prägnante Anknüpfung im Maßgeflecht des Bauwerkes" gelten als die wichtigeren Regeln.

48 Dass diese Prämisse berechtigt ist, werden die weiteren Recherchen über die tiefenplanerische Verflochtenheit der angenommenen Schale mit dem Maßgerüst des Bauwerks zeigen. 
wir den Hofoktogonradius $r_{h}$ in zwei Abschnitte a und b so zu teilen, dass die Teillängen jeweils in Einheiten von $\phi$ und Palm ganze oder zumindest halbe Maßzahlen ergeben (Abb. 11). Die ursprüngliche Wasserschale soll monolithisch gewesen sein. ${ }^{49} \mathrm{Um}$ uns von der praktisch herstellbaren Größe einer monolithischen Wasserschale nicht allzu weit zu entfernen, berechnen wir die plankonformen Schalengrößen bis a $=3,42 \mathrm{~m}=8$, was eine Schalendiagonale von ca. 6,8 m bedeuten würde (Tab. 2). Durch die Überprüfung der Paritäten in Halb-Palm-Schritten und $\phi$ stellen sich zwei Zahlen heraus, die unsere Bedingungen erfüllen könnten. Diese sind 6,50 und 13,00 Palm, welche den Maßzahlen 4,01 und 8,03 in ф-Einheit entsprechen (Tab. 2).

$\begin{array}{rrrrrrrrrr}\mathrm{P} & 5,00 & 5,50 & 6,00 & 6,50 & 7,00 & 7,50 & 8,00 & \ldots & \ldots \\ \phi & 3,09 & 3,40 & 3,70 & 4,01 & 4,32 & 4,63 & 4,49 & \ldots & \ldots \\ & & & & & & & & & \\ \mathrm{P} & \ldots & \ldots & 11,00 & 11,50 & 12,00 & 12,50 & 13,00 & 13,50 & 14,00 \\ \phi & \ldots & \ldots & 6,80 & 7,11 & 7,42 & 7,73 & 8,03 & 8,34 & 8,65\end{array}$

Tab. 2: Berechnung des Brunnenschalenradius $r_{s}$ nach planimmanenten Regeln.

Die Entscheidung zwischen den beiden Schalengrößen fällt aus folgenden Gründen zugunsten der kleineren Schalenabmessung aus.

- Die Herstellung und der Transport einer monolithischen Brunnenschale von knapp 7 m (oder 13 x 2=26 p) Durchmesser für die größere Lösung wäre im 13. Jahrhundert kaum möglich gewesen.

- Die Wasserhaltung dieser Brunnenschale mittels Zisterne - bei beachtlichen Verdunstungsmengen im Sommer - wäre kaum aufrecht zu erhalten gewesen.

Dagegen ist die kleinere Alternative, nämlich eine Brunnenschale mit Schalenoktogonradius $r_{s}=4 \phi(=1,71 \mathrm{~m})$, nicht nur aus erwähnten Überlegungen zu bevorzugen, sondern auch aus folgenden metrologischen Gesetzmäßigkeiten.

- Ein Schalenradius $r_{s}=4,0$ ф (Abb. 11) ergibt eine Schalenfläche $F_{s}=53$ $(\mathrm{Phi})^{2}{ }^{20}$ Diese Maßzahl knüpft sich durch die Stauferzahl 8 an die ursprüngliche Trapezraumfläche $\mathrm{F}_{\mathrm{t}}=423,6(\mathrm{Phi})^{2}$. Denn

$$
423,6: 53=7,99 \sim 8
$$

- Außerdem ist die Quersumme von 53 ebenfalls 8.

- Der Hofradius $r_{h}=21 \phi$ wird durch den Schalenradius $r_{s}=a=4 \phi$ im Verhältnis des halben Goldenen Schnittes geteilt (Abb. 11).

$$
\text { b: }(a+b)=17: 21=0,809 \times 2=1,618=\phi .
$$

49 Legler (wie Anm. 1), S. 13.

50 Vgl. Anm. 17. 
- Außerdem gilt

$$
\mathrm{b}: \mathrm{a}=17: 4=4,25=\phi \times \mathrm{x} \text { x } \phi, \operatorname{mit}(\Delta=0,3 \%),{ }^{51}
$$

was die Trinität assoziiert.

- Der Wasserschalenradius $\overline{\mathrm{OA}}=\mathrm{a}$ samt Innenhofbreite $\overline{\mathrm{AB}}=\mathrm{b}$ (Abb. 5) sind metrologisch markant mit der Innenhofmauerstärke $\overline{\mathrm{BC}}$ verknüpft:

$$
\begin{aligned}
& \overline{\mathrm{OA}}+\overline{\mathrm{AB}}=4+17=21 \\
& \overline{\mathrm{OB}}: \overline{\mathrm{BC}}=21: 5,5=3,82,
\end{aligned}
$$

wobei $3,82: 10=0,382=\phi^{-2}$ und damit als Reihenglied der geometrischen Progression des Goldenen Schnitts dargestellt wird (Anm. 16, Abb. 4).

Das gleiche Verhältnis lässt sich übrigens auch für die Trapezraumbreite $\overline{\mathrm{CD}}$ samt Außenmauerstärke $\overline{\mathrm{DE}}$ zu der Innenhofmauerstärke $\overline{\mathrm{BC}}$ herstellen (Abb. 5)

$$
(\overline{\mathrm{CD}}+\overline{\mathrm{DE}}): \overline{\mathrm{BC}}=(15+6): 5,5=3,82 .
$$

Die größte Abmessung der Schale ist jedoch die Oktogonaldiagonale $\mathrm{d}=2 \mathrm{x}$ $\mathrm{R}_{\mathrm{s}}$ (Abb. 11). Mit Hilfe der mathematischen Tabellen errechnet man

$$
\begin{aligned}
& d=2 \cdot r_{s}: 0,924 \phi \\
& \text { d.h. } \\
& d=8,66, \phi=14 \text { und } p=3,68 \mathrm{~m} .
\end{aligned}
$$

Mit dem dargelegten dichten Verknüpfungsnetz der Abmessungen in der Planung kann man davon ausgehen, dass eine originalgetreue Nachbildung der Brunnenschale in Castel de Monte grundsätzlich möglich ist.

\section{Typisierung des Burgenbaus}

Die immensen kreativen Anstrengungen, die für die Erstellung eines Bauplans mit austauschbaren Maßeinheiten - ohne Beeinträchtigung seiner Merkmale in Castel del Monte unternommen wurden, sind nicht zuletzt bedingt durch die Einführung des abstrakten Maß-Rahmens $\phi$. Da erst durch diesen Kunstgriff die Variabilität des Baumaßes, bzw. die Typisierung des Bauplans möglich wird, könnte man davon ausgehen, dass die Konzeption eines Typenplans für den Burgenbau durchaus beabsichtigt war; zumal man sich in der historischen Hauptphase des Burgenbaus befand. ${ }^{52}$ Schließlich ist bekannt, dass in den mittelalterlichen Bauhütten bereits typisierte Figuren, Ornamente, technische Geräte und anderes mehr hergestellt wurden.

Das kaiserliche Ansinnen, den Burgenbau zu typisieren, wäre vor allem in seiner wahren Besessenheit des Bauens begründet, die zu einem gigantischen

51 Vgl. Anm. 19.

52 Maurer (wie Anm. 34), S. 121. 

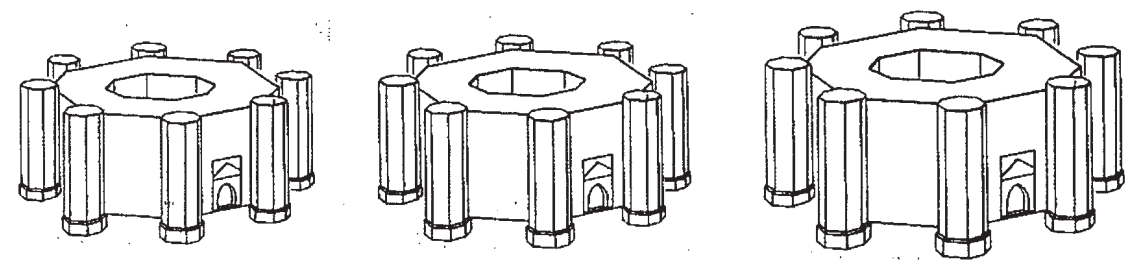

Abb. 12: Beispiele für typisierte Kastellgrößen. Quelle: Eigene Zeichnung.

Ausmaß der Bautätigkeit führte. ${ }^{53}$ Dies stand aber auch mit seiner Machtpolitik im Einklang. Schließlich hat er in knapp drei Dezennien in seinem Königreich Sizilien mehr als 200 Profanbauten errichtet. ${ }^{54}$ In dem Bauplan Castel del Montes werden alle Kriterien berücksichtigt, die aus der Sicht Friedrichs II. ein typisierter Burgbauplan enthalten musste. Während die Außenansicht der Burg von acht oktogonalen Türmen als staufische Sinnbilder bestimmt wird, haben die inneren Räumlichkeiten durch mystische Chiffrierung eine diskrete christliche Glaubensbezeugung und Aura der Frömmigkeit. ${ }^{55}$ Zudem ist das Baumaß austauschbar und variabel zu gestalten, wodurch unterschiedliche Burggrößen gebaut werden können (Abb. 12).

\section{Abschließende Betrachtung}

Die Zielsetzung dieser Untersuchung war - wie bereits in der Einleitung erwähnt - den historischen Spuren der zweiten Lesart des Goldenen Schnitts nachzugehen. Das Beispiel Castel del Monte ist zwar ein bedeutsamer Meilenstein in diesem Entwicklungsverlauf, wirft aber wiederum die Frage nach dessen Vorgeschichte auf. Denn auch der Kastellplan dürfte nicht ohne inspirierende Exempel entstanden sein. In der Tat kann man bereits in der Antike, etwa im Athenatempel (Paestum), gleichartige Anwendungen des formalen Zahlenwerts vom Goldenen Schnitt finden. ${ }^{56}$ In Isfahan hat man den $\phi$-Wert als Maßfaktor angewandt. ${ }^{57} \mathrm{Nach}$ den Beispielen in Castel del Monte und

53 Carl A. Willemsen u. Dagmar Odenthal, Apulien, Köln 1958, S. 47 f.

54 Götze (wie Anm. 1), S. 29.

55 Schaller (wie Anm. 20), S. 596.

56 Im Athenatempel in Paestum ist der formale Zahlenwert des Goldenen Schnitts $(\phi=8 / 5$ $=1,6)$ als Planmaß für den Entwurf der Stylobat zugrunde gelegt worden. Das Baumaß bleibt aber - ähnlich wie im Fall von Castel del Monte - ein konkretes Maß; nämlich der dorisch-pheidonische Fuß $(=32,8 \mathrm{~cm})$. Der Säulenabstand von $8 \mathrm{Fu} \beta$ entspricht dann 5 ф; vgl. Naredi-Rainer (wie Anm. 11), S. 153.

57 Im Mittelalter ist bei der Normung der Bauelle (Zar'e) der Kehrwert von $\phi(=1,6)$, d.h. 25/40, benutzt worden. Vgl. Mehdi Nadji, Die Dreiunddreißigbogenbrücke in Isfahan: Metrologisches Normsystem und Symbol für Staatsdoktrin, in: architectura 33, 2003, S. 175-188, hier S. 187. 
in Isfahan begegnen wir in der Gegenwart dem Modulor als Maschennetz, in dem Le Corbusier ebenfalls mit dem formalen Zahlenwert operiert hat. ${ }^{58}$

Diese vereinzelten Beispiele lassen die Annahme zu, dass der formale Zahlenwert des Goldenen Schnitts des Öfteren als Quasimaß benutzt worden sein dürfte. Die ausführliche und objektbezogene Untersuchung dieser abstrakten Maßauffassung im bauhistorischen Kontext bedarf aber noch besonderer Untersuchungen.

Anschrift des Verfassers: Dr.-Ing. Mehdi Nadji, Mainstr. 38, D-50996 Köln 